\title{
Comparative Analysis of Low Leakage SRAM Cell at 32nm Technology
}

\author{
Jaspreet Kaur \\ Electronics and Communication Engg Section \\ Yadavindra College of Engineering, \\ Talwandi Sabo, India
}

\author{
Candy Goyal \\ Assistant Professor, Electronics and \\ Communication Engg Section \\ Yadavindra College of Engineering, \\ Talwandi Sabo, India
}

\begin{abstract}
Continuous scaling of the transistor size and reduction of the operating voltage has led to a significant performance improvement of integrated circuits. Low power consumption and smaller area are the most important criteria for the fabrication of DSP systems. Static random access memories (SRAMs) consist of almost $90 \%$ of very large scale integrated (VLSI) circuits. The ever-increasing demand for larger data storage capacity has driven the fabrication technology and memory development toward more compact design rules and, consequently, toward higher storage densities. This paper deals with design of low power static random-access memory (RAM) cells and peripheral circuits for standalone RAMs, in $32 \mathrm{~nm}$ focusing on stable operation and reduced leakage power dissipation. The work is carried out on Tanner Tool version 13 at $32 \mathrm{~nm}$ technology.
\end{abstract}

\section{Keywords}

SRAM, VLSI, Leakage Power Dissipation, Static Power, Dynamic Power.

\section{INTRODUCTION}

Semiconductor memory arrays capable of storing large quantities of digital information (binary logic ' 1 ' and ' 0 ' bits) are essential to all digital systems. SRAM (Static RAM) is random access memory (RAM) that retains data bits in its memory as long as power is being supplied. Therefore SRAM is a type of volatile semiconductor memory. Unlike dynamic RAM (DRAM), bits are stored in cells consisting of a capacitor and a transistor. SRAM cell consists of a bi-stable latching circuitry made of Transistors/MOSFETS to store each bit, therefore, the cell data is kept as long as the power is turned on and it does not have to be periodically refreshed. SRAM is mainly used for the cache memory in microprocessors, laptops \& hand held devices due to high speed and low power consumption. The leakage power affects the chip design process. Speed of SRAM and Power consumption should be taken care of for designing a chip. In SRAM the data is lost when the memory is not electrically powered. SRAM is faster and more reliable than the DRAM. Unfortunately, it is much more expensive to produce than DRAM. Due to its high cost, SRAM is often used only as a memory cache. An SRAM is designed for two needs: For a direct interface with the CPU at speeds not attainable by DRAMs and to replace DRAMs in systems that require very low power consumption. In the first role, the SRAM serves as cache memory, interfacing between DRAMs and the CPU.

The second role for SRAM technology is low power applications. In this case, SRAMs are used in most portable equipment because the DRAM refresh current is several orders of magnitude more than the low-power SRAM standby current. Most of industrial and scientific subsystems, automotive electronics, digital cameras, cell phones and synthesizers contain Static RAM.

SRAM is also used in Cable Modems, workstations, routers, personal computers and peripheral equipment, internal CPU caches and external burst mode SRAM caches, hard disk buffers, etc. LCD screens and printers also employ SRAM to hold the image printed or to be displayed. SRAM buffers are also found in CDROM and CDRW drives to buffer track data, which is transferred in blocks instead of single values.

\section{TECHNIQUES FOR OPTIMIZATION OF LEAKAGE POWER USING 1-BIT SRAM CELLS}

W/L Ratios for PMOS \& NMOS

Equation (1) gives the (W/L) ratio for PMOS transistor [20].

$$
\frac{\left(\frac{W}{L}\right)_{s}}{\left(\frac{W}{L}\right)_{3}}<\frac{\mu_{n}}{\mu_{p}} \cdot \frac{2\left(V_{D D}-1.5 V_{T, n}\right) V_{T, n}}{\left(V_{D D}+V_{T, p}\right)^{2}}
$$

Equation (2) gives the (W/L) ratio for NMOS transistor [20].

$$
\begin{gathered}
\frac{k_{n, 3}}{k_{n, 1}}=\frac{\left(\frac{W}{L}\right)_{3}}{\left(\frac{W}{L}\right)_{1}}<\frac{2\left(V_{D D}-1.5 V_{T, n}\right) V_{T, n}}{\left(V_{D D}-2 V_{T, n}\right)^{2}} \\
\mathrm{~W}_{1}=\mathrm{W}_{\text {NMOS }}=5 \times \mathrm{L} \\
\mathrm{W}_{3}=\mathrm{W}_{\text {ACCESS }}=2.5 \times \mathrm{L} \\
\mathrm{L}=32 \mathrm{~nm} \\
\mathrm{~W}_{5}=\mathrm{W}_{\text {PMOS }}=1.78 \times \mathrm{W} \\
\mu_{\mathrm{n}} / \mu_{\mathrm{p}}=2
\end{gathered}
$$

From the above equations we get:

$$
\begin{aligned}
& \mathrm{W}_{\text {PMOS }}=142 \mathrm{~nm} \\
& \mathrm{~W}_{\text {ACCESS }}=80 \mathrm{~nm} \\
& \mathrm{~W}_{\text {NMOS }}=160 \mathrm{~nm}
\end{aligned}
$$

\subsection{Conventional 6T SRAM Cell}

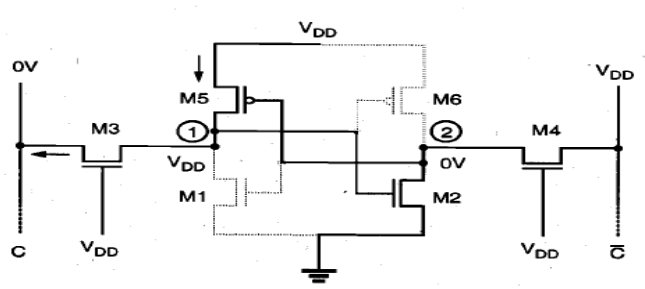

Figure 1.Conventional 6T SRAM Cell 
Above Figure shows the write mode of conventional SRAM cell. Word line is used for enabling the access transistors M3 and M4 for write operation.

BL and BLB lines are used to store the data and its compliment. For write operation one bit line is High and the other bit line is on low condition.

For writing "0" BL is High and BLB is low. When we assert the word line high transistor M2 and M4 are ON and any charged stored in the BLB goes through M4- M2 path to ground.

Due to Zero value at Q the M5 transistor is ON and M1 is OFF so the charged stored at $\mathrm{Q}$ line.

Similarly in the write "1" operation BLB is high due to this M1 is $\mathrm{ON}$ and the charge store on BL is discharged through the M3-M1 path to ground and due to this low value on Q the M6 is $\mathrm{ON}$ and $\mathrm{M} 2$ is OFF so the charged stored on the QB

\subsection{P-P-N Based Differential 10T SRAM Cell}

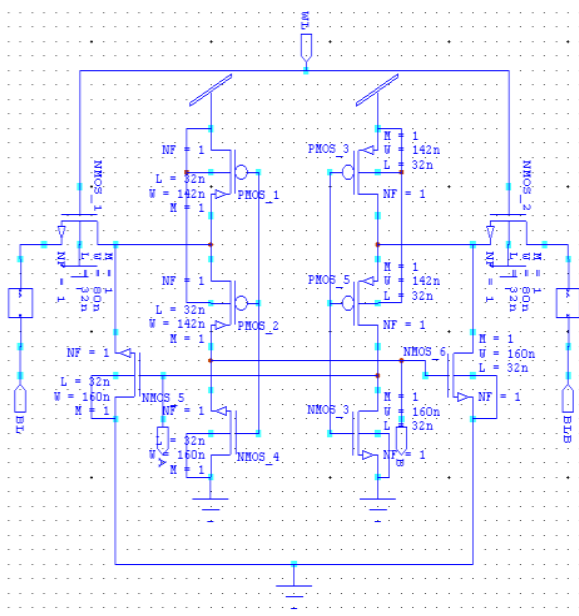

Figure 2. Schematic of P-P-N Based Differential 10T SRAM Cell

As shown in Figure 2. In addition to the standard 6T SRAM cells one extra signal called VGND, denoting a virtual ground signal is added. This cell is called P-P-N based since each of three transistors cascaded in a P-P-N sequence from top to bottom. Stack transistors are connected in pull down paths to reduce power dissipation [2].

\subsection{Fully Differential Based 10T SRAM Cell}

Design of cell consists series connection of tail transistor, Gate electrode is controlled by output of XOR gate. Tail transistor and XOR gate is shared by all the cells in a row. Inputs are tapped from WWL and RWL. Width of the Tail Transistor is kept large $(800 \mathrm{~nm})$ in order to Improve Static Noise Margin, resulting in stability [2].

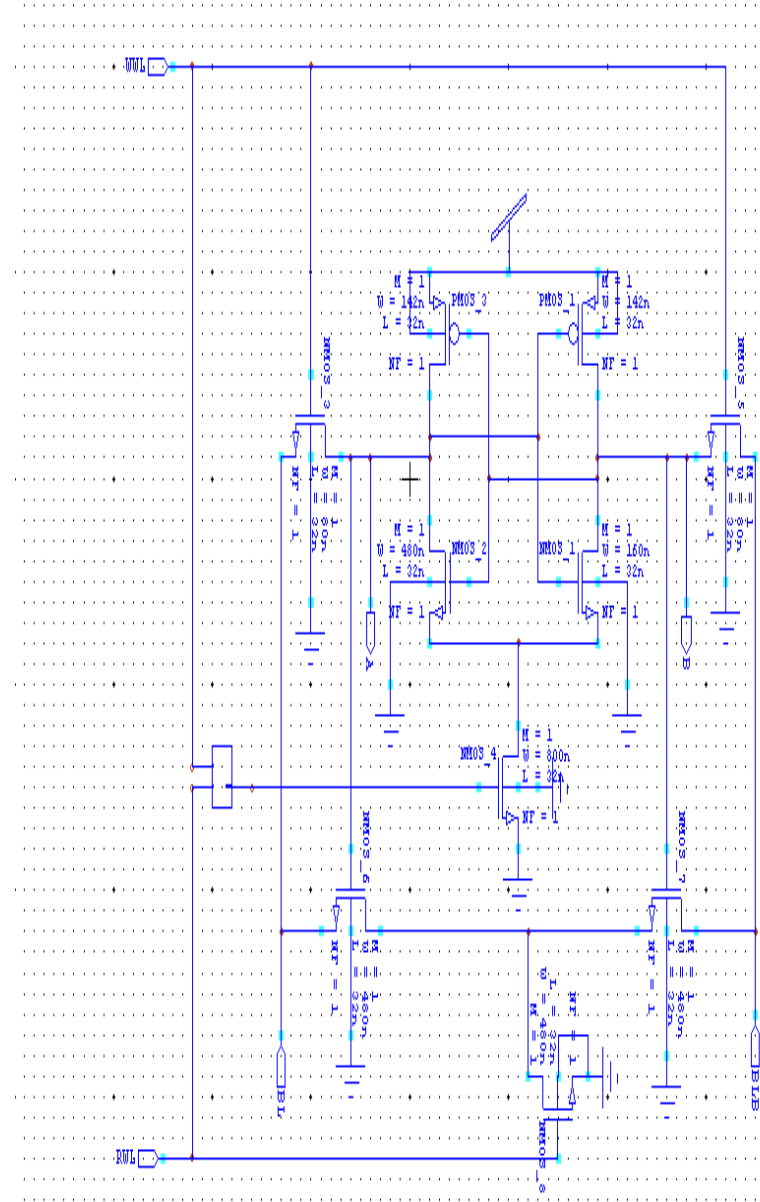

Figure 3. Schematic of Fully Differential Based 10T SRAM Cell

\subsection{Anti-leakage Based 10T SRAM Cell}

In this low power technique, the decoupling transistor P3, P4 and the anti-leakage transistor P5, P6 reduce the leakage power. The decoder section can also be designed for high $\mathrm{V}_{\text {th }}$ operation, by which the leakage currents can be reduced [2].

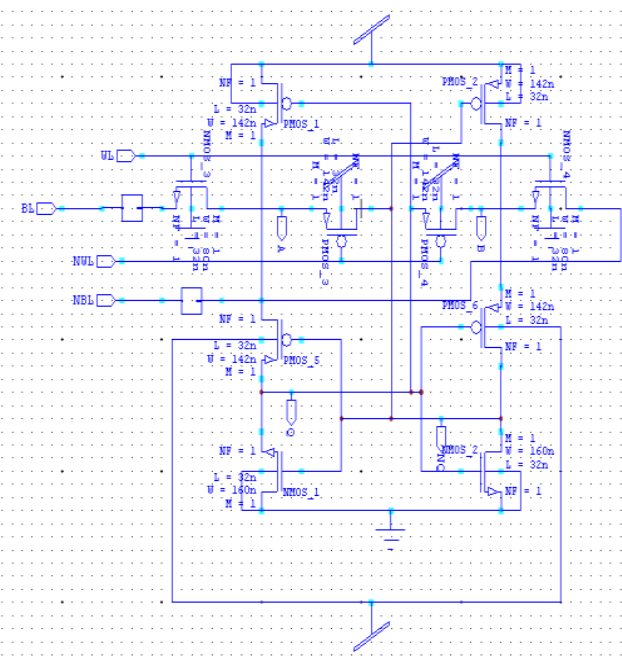

Figure 4.Schematic of Antileakage Based 10T SRAM Cell 


\section{MODIFIED 1-BIT SRAM CELLS}

\subsection{NMOS SRAM Cell}

The NMOS SRAM Cell has been implemented using two NMOS transistors with the driver transistors as shown in Figure.5.

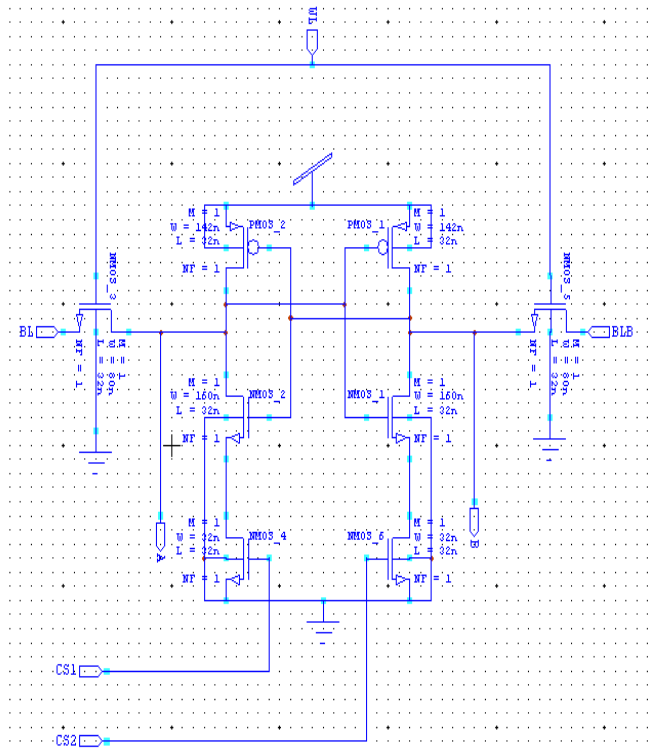

Figure 5. Schematic of Modified 1-BIT NMOS SRAM Cell

\subsection{P-NMOS SRAM Cell}

The P-NMOS SRAM Cell has been implemented using one PMOS transistor and one NMOS with the driver transistors as shown in Figure.6.

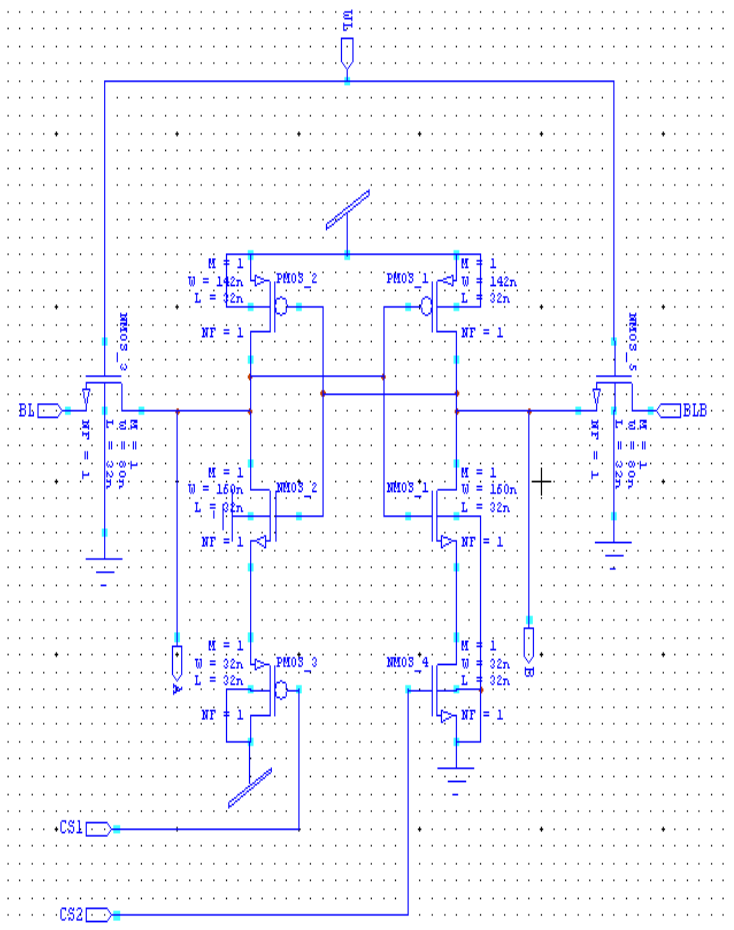

Figure 6. Schematic of Modified 1-BIT P-NMOS SRAM Cell

\subsection{N-PMOS SRAM Cell}

The N-PMOS SRAM Cell has been implemented using one NMOS transistor and one PMOS with the driver transistors as shown in Figure.6.

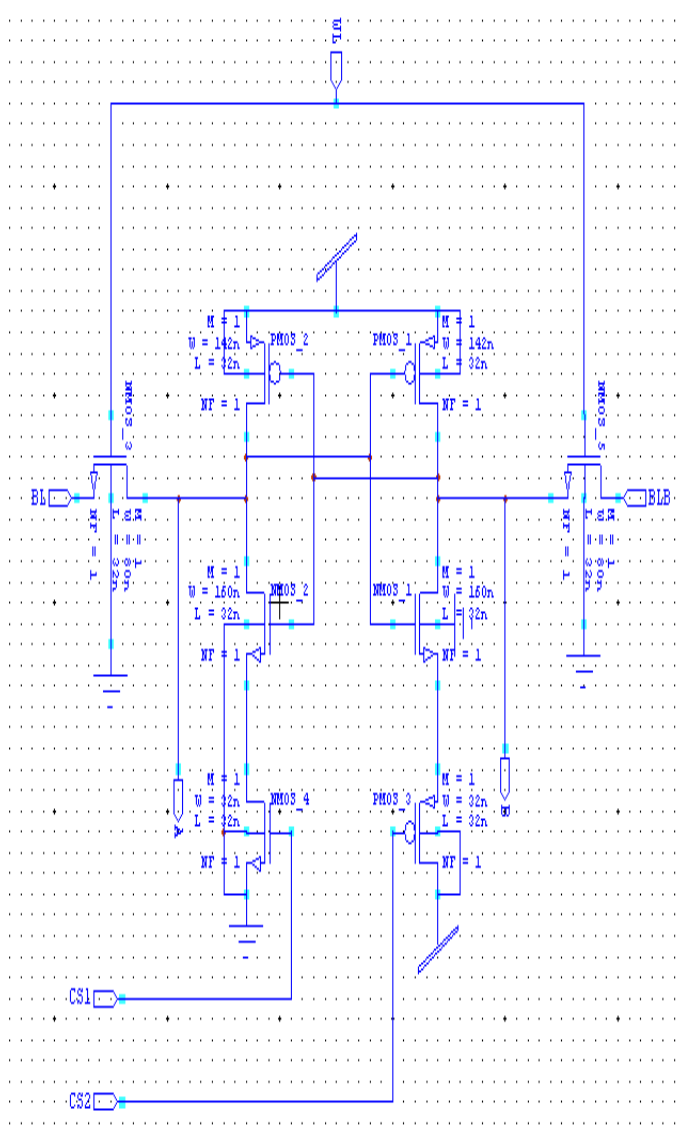

Figure 7. Schematic of Modified 1-BIT N-PMOS SRAM Cell

\section{SRAM ARCHITECTURE}

Figure 8. Gives an overview of an SRAM memory design for a single data bit input / output. A single address of $N+M$ bits is split into $\mathrm{N}$ row addresses and $\mathrm{M}$ column addresses. The row address is first decoded, so that one out of $2^{N}$ word lines in the memory array (of size $2^{N} \times 2^{M}$ ) is being selected. Most RAMs are built so that all cells in the same row are activated. Consequentially, all $2^{M}$ bit line pairs forming the columns leaving the memory array now contain data. The column decoder select one of these line pairs based on the column address. Typically, the signal is sense-amplified, and then put into the data read buffer.

The main SRAM building blocks are as follows.

- $\quad$ SRAM cell

- Pre-Charge Circuit

- Column decoder Circuit

- Sense Amplifier

- Row decoder 


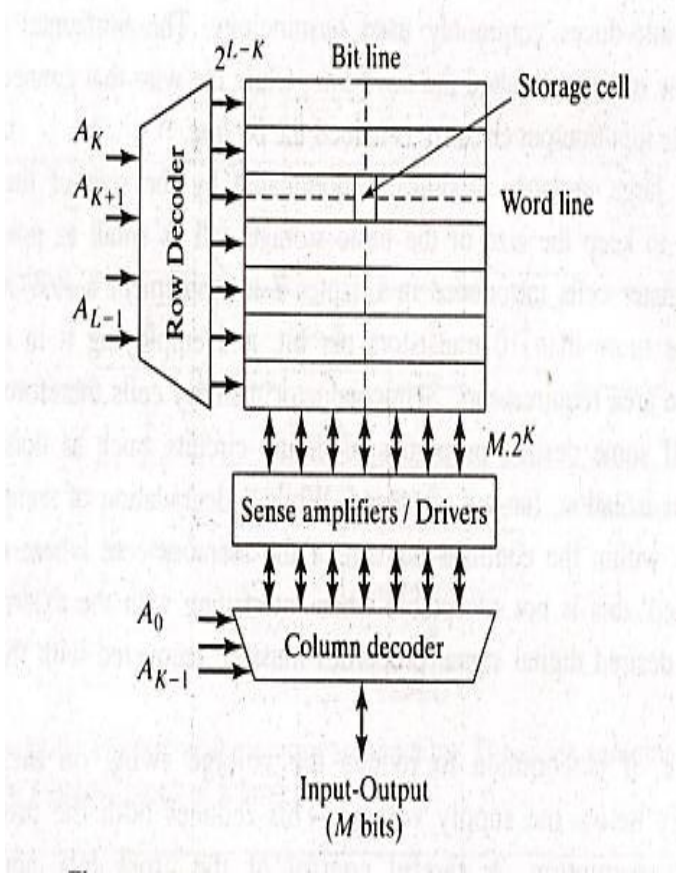

Figure 8. SRAM Architecture

\subsection{Pre-Charge Circuit}

The pre-charge circuitry brings the BL and BLB to be the same voltage before a read cycle. The simplest version of pre-charge circuitry consists of three transistors that can be either NMOS or PMOS devices. Two of the transistors are used to connect the bit lines to $\mathrm{V}_{\mathrm{dd}}$, and the third transistor is connected between the two bit lines to ensure that the lines end up being the same voltage. To pre-charge the bit lines, the signal PRE is brought to logic 0 . This turns on all three transistors which then charge and equalize BL and BLB to be $\mathrm{V}_{\mathrm{dd}}$.

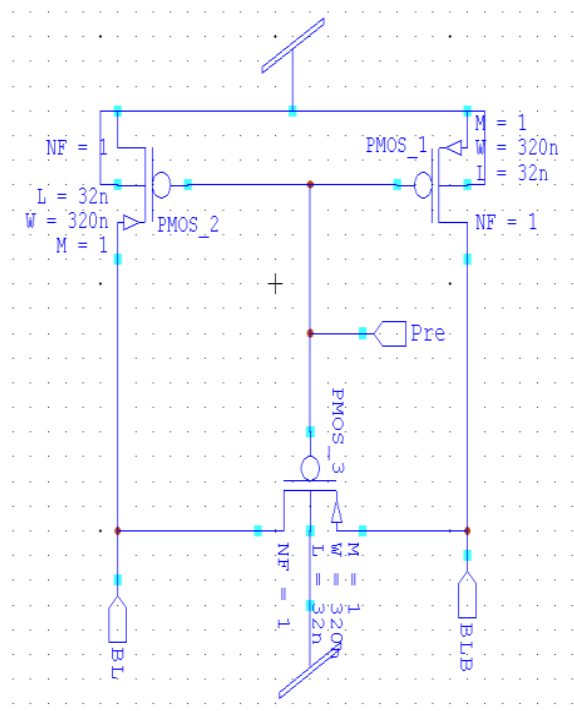

Figure 9. Schematic of Pre-Charge Circuit

\subsection{Column/Row Decoder}

A decoder has 16-line de-multiplexing capability which decodes 4 binary-coded inputs into one of 16 mutually exclusive outputs.

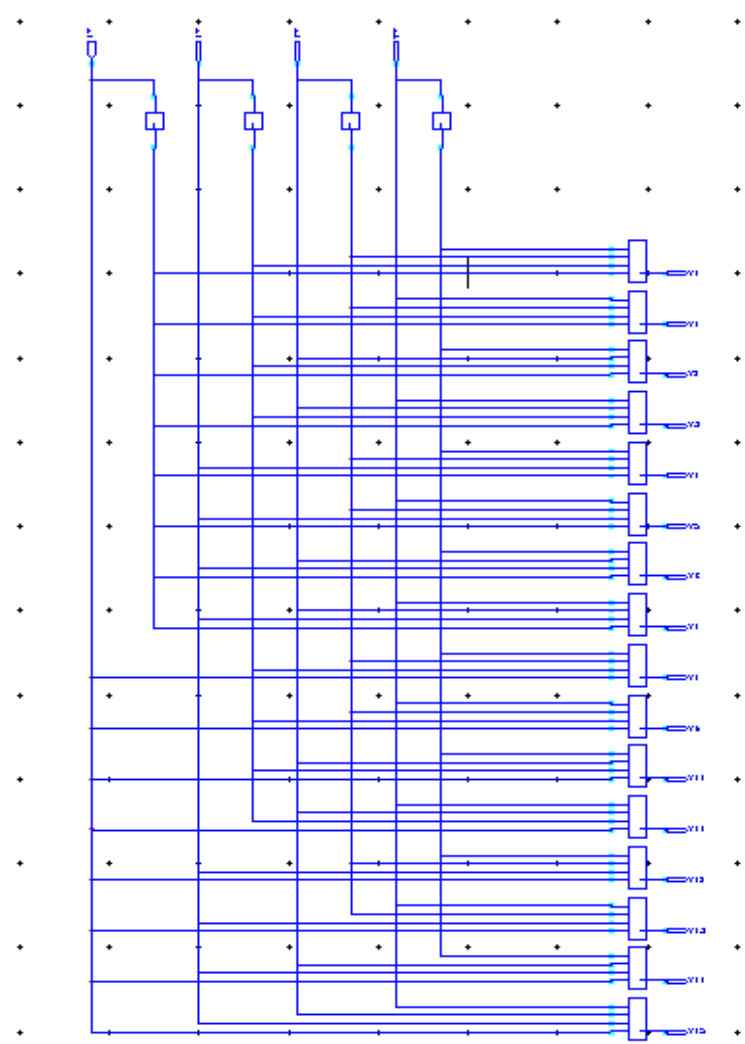

Figure 10. Schematic of $4 \times 16$ Decoder Circuit

\subsection{Sense Amplifier Circuit}

The sense amplifier is in charge of detecting what value is stored in an SRAM cell during a read cycle and displaying that value at the output. Since only one row of data is accessed during each read cycle, each column of cells within the SRAM array requires only one sense amplifier. A sense amplifier works by sensing a relatively small difference between the voltages of the two bit lines, then amplifying the difference at the output to show if a cell is storing either logic 1 or 0 . The bit lines are pre-charged before each read cycle to ensure that the difference between the bit line voltages are caused by the value that is stored in the cell. If the bit lines are not precharged, there is a chance that the sense amplifier could misread and present an incorrect value at the output. 


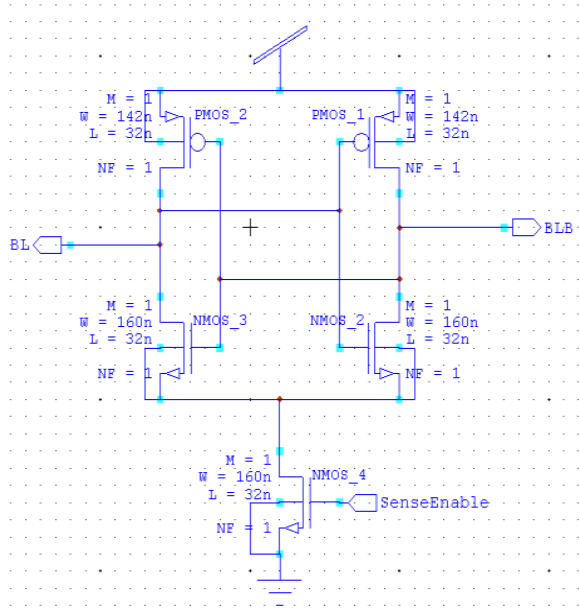

Figure 11. Schematic of Sense Amplifier Circuit

\subsection{6x16 SRAM ARRAY}

The above 1 Bit building blocks are used to design the $16 \times 16$ SRAM Array.

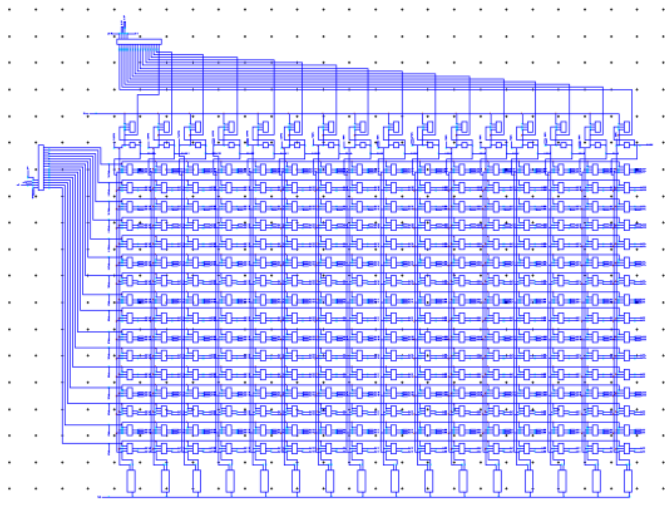

Figure 12 a. 6T SRAM 16x16 Array

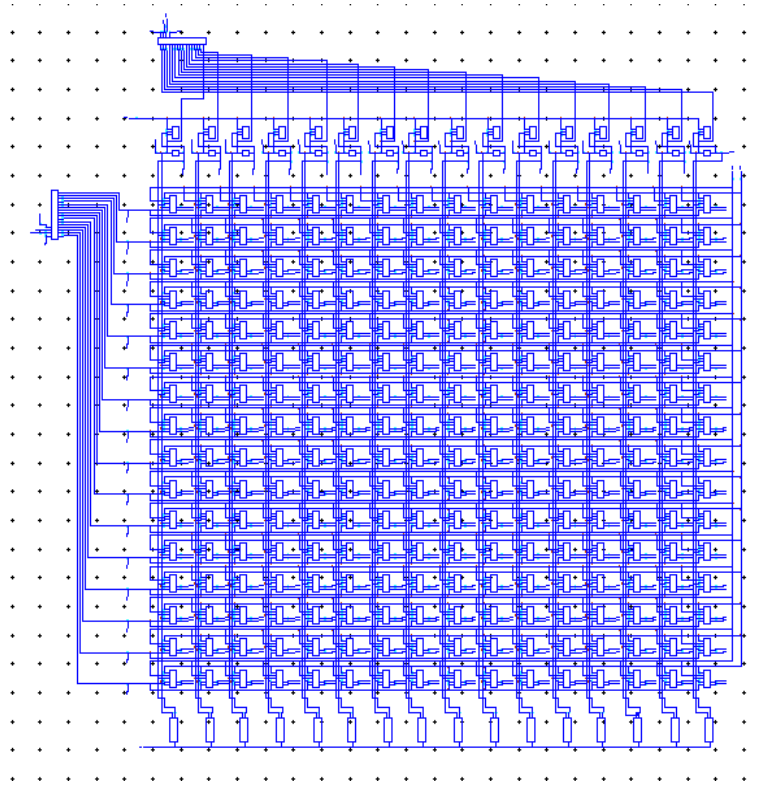

Figure 12.b Modified NMOS SRAM 16x16 Array

\section{STABILITY}

The first consideration in the SRAM cell design is the stability that is the ability to hold a stable cell state.

Static Noise Margin (SNM) is an important parameter in determining the cell stability. The SNM of SRAM cell is defined as the maximum value of noise that can be tolerated by the cross-coupled inverters before altering state.

According to the results shown in the SNM of the conventional SRAM cell ( $\left.\mathrm{SNM}_{\text {conv }}\right)$ is characterized with the following expressions.

$$
\begin{aligned}
\mathrm{SNM}_{\mathrm{Conv}}= & V_{T}-\frac{1}{k+1} \\
& \cdot\left(\frac{V_{\mathrm{DD}}-\frac{2 r+1}{r+1} V_{T}}{1+\frac{r}{k(r+1)}}-\frac{V_{\mathrm{DD}}-2 V_{T}}{1+k \frac{r}{q}+\sqrt{\frac{r}{q}\left(1+2 k+\frac{r}{q} k^{2}\right)}}\right) \\
\text { where } & =\beta_{\text {driver }} / \beta_{\text {access; }} ; \\
r & =\beta_{\text {load }} / \beta_{\text {access }} ; \\
q & =(r / r+1)\left(\sqrt{\left(r+1 /\left(r+1-V_{s}^{2} / V_{r}^{2}\right)\right)}-1\right) ; \\
V_{s} & =V_{\mathrm{DD}}-V_{T} ; \\
V_{r} & =V_{s}-r /(r+1) V_{T} .
\end{aligned}
$$

$\beta_{\text {driver, }} \beta_{\text {access }}$ and $\beta_{\text {load }}$ are the $\mathrm{W} / \mathrm{L}$ ratios of driver transistors $(\mathrm{N} 1, \mathrm{~N} 2)$, load transistors $(\mathrm{P} 1, \mathrm{P} 2)$ and access transistors $(\mathrm{N} 3$, $\mathrm{N} 4)$, respectively $\mathrm{V}_{\mathrm{DD}}=1.0 \mathrm{v}, \mathrm{V}_{\mathrm{T}}=0.4 \mathrm{v}$.

For Fully Differential 10T SRAM Cell the cell ratio of the ZA cell having tail transistor $\mathrm{N} 4$ is $\mathrm{r}^{\prime}=\left(\beta_{\mathrm{N} 2} \times \beta_{\mathrm{N} 4}\right) /\left(\beta_{\mathrm{N} 1}\left(\beta_{\mathrm{N} 2}+\right.\right.$ $\left.\left.\beta_{\mathrm{N} 4}\right)\right)$. To maintain the $\left(\mathrm{SNM}_{\mathrm{ZA}}\right)$ same as the $\left(\mathrm{SNM}_{\text {Conv }}\right)$ the cell ratio r' must be equal to the conventional cell ratio $r$, which can be achieved by appropriate choice of $\beta_{\mathrm{N} 2}$ and $\beta_{\mathrm{N} 4}$.

The SNM $\mathrm{ZA}_{\mathrm{Z}}$ in different combinations of $\beta_{\mathrm{N} 2}$ and $\beta_{\mathrm{N} 4}$ when $\beta_{\mathrm{N} 1}=1$ keeping the value of $\beta_{\mathrm{N} 2}$ and $\beta_{\mathrm{N} 4} \quad 3$ and 5 (i.e., the conventional cell ratio is fixed to 2 ).

The ZA cell ratio $r^{\prime}=15 / 8$ approximates the conventional cell ratio $\mathrm{r}=2$ that verifies the estimation model developed by Keeping the value of $\mathrm{W}_{\mathrm{N} 1}, \mathrm{~W}_{\mathrm{N} 2}$ and $\mathrm{W}_{\mathrm{N} 4}$ (tail transistor) $160 \mathrm{~nm}$, $480 \mathrm{~nm}$ and $800 \mathrm{~nm}$.

Case I: Calculation of SNM, without taking the average of the widths of Driver and Load Transistors.

Case II: Calculation of SNM, by taking the average of the widths of Driver and Load Transistors.

Table 1. SNM of 1-Bit SRAM Cells

\begin{tabular}{|c|c|c|c|}
\hline Sno. & SRAM Cell (1 Bit) & $\begin{array}{c}\text { Static Noise } \\
\text { Margin (mV) } \\
\text { Case I }\end{array}$ & $\begin{array}{c}\text { Static Noise } \\
\text { Margin } \\
(\mathrm{mV}) \text { Case II }\end{array}$ \\
\hline 1. & Conventional 6T & 434 & - \\
\hline 2. & PPN 10T & 434 & 421 \\
\hline 3. & $\begin{array}{c}\text { Fully Differential } \\
\text { 10T }\end{array}$ & 437 & - \\
\hline 4. & Anti-Leakage 10T & 434 & 436 \\
\hline 5. & Modified NMOS & 434 & 427 \\
\hline 6. & Modified P-NMOS & 434 & 427 \\
\hline 7. & Modified N-PMOS & 434 & 427 \\
\hline
\end{tabular}




\section{RESULTS AND COMPARISON}

This section provides the detailed simulation analysis of the different 1 bit and 16x16 bit configurations of SRAM cell. The Average Power Dissipations (Static + Dynamic), Delay and Static Noise Margin for SRAM cell are estimated. The schematic of the SRAM cell is designed and implemented by using Tanner Tool V13.Simulation has been done in $32 \mathrm{~nm}$ environment with a power supply of 1.0 volt and at an operating frequency of $500 \mathrm{MHz}$

The simulation results for 1 Bit and $16 \times 16$ Bit SRAM Cells is shown in Table 2 and Table 3 Respectively.

Table 2. Comparison of 1-Bit SRAM Cells in terms of Power \& Delay

\begin{tabular}{|c|c|c|c|c|c|}
\hline Sno. & $\begin{array}{c}\text { SRAM Cell } \\
(1 \text { Bit })\end{array}$ & $\begin{array}{c}\text { Average } \\
\text { Power } \\
(\mu \mathrm{W})\end{array}$ & $\begin{array}{c}\text { Leakage } \\
\text { Power } \\
(\mu \mathrm{W})\end{array}$ & $\begin{array}{c}\text { Delay } \\
(\mathrm{ns})\end{array}$ & $\begin{array}{c}\text { PDP } \\
(\mathrm{fj})\end{array}$ \\
\hline 1. & $\begin{array}{c}\text { Conventional } \\
\text { 6T }\end{array}$ & 2.64 & 0.20 & 0.06 & 0.15 \\
\hline 2. & PPN 10T & 83.7 & 78.9 & 0.63 & 52.7 \\
\hline 3. & $\begin{array}{c}\text { Differential } \\
10 \mathrm{~T}\end{array}$ & 12.0 & 7.90 & 0.06 & 0.72 \\
\hline 4. & $\begin{array}{c}\text { Anti- } \\
\text { Leakage 10T }\end{array}$ & 51.6 & 0.39 & 0.09 & 4.64 \\
\hline 5. & $\begin{array}{c}\text { Modified } \\
\text { NMOS }\end{array}$ & 0.90 & 0.19 & 0.05 & 0.04 \\
\hline 6. & $\begin{array}{c}\text { Modified P- } \\
\text { NMOS }\end{array}$ & 6.27 & 10.9 & 0.05 & 0.31 \\
\hline 7. & $\begin{array}{c}\text { Modified N- } \\
\text { PMOS }\end{array}$ & 6.27 & 0.16 & 0.04 & 0.25 \\
\hline
\end{tabular}

Table 3. Comparison of 16x16 Bit SRAM Cells in terms of Power \& Delay

\begin{tabular}{|c|c|c|c|c|c|}
\hline Sno. & $\begin{array}{c}\text { SRAM Cell } \\
(16 x 16 \text { Bit })\end{array}$ & $\begin{array}{c}\text { Average } \\
\text { Power } \\
(\mathrm{mW})\end{array}$ & $\begin{array}{c}\text { Leakage } \\
\text { Power } \\
(\mathrm{mW})\end{array}$ & $\begin{array}{c}\text { Delay } \\
(\mathrm{ns})\end{array}$ & $\begin{array}{c}\text { PDP } \\
(\mathrm{pj})\end{array}$ \\
\hline 1. & $\begin{array}{c}\text { Conventional } \\
6 \mathrm{~T}\end{array}$ & 6 & 5.9 & 0.29 & 1.74 \\
\hline 2. & PPN 10T & 21.5 & 21.5 & 0.39 & 8.38 \\
\hline 3. & $\begin{array}{c}\text { Differential } \\
10 \mathrm{~T}\end{array}$ & 12.8 & 14.3 & 0.81 & 10.3 \\
\hline 4. & $\begin{array}{c}\text { Anti- } \\
\text { Leakage 10T }\end{array}$ & 3.95 & 2.12 & 0.41 & 1.61 \\
\hline 5. & $\begin{array}{c}\text { Modified } \\
\text { NMOS }\end{array}$ & 1.79 & 2.06 & 0.03 & 0.05 \\
\hline
\end{tabular}

\section{CONCLUSION \& FUTURE SCOPE}

Low leakage SRAM Cells using Tanner Tool (v.13) is designed in this paper. Three Modified Low Power NMOS SRAM cell has been presented in this work. In the Modified SRAM cell average power dissipation is reduced because of the reduction of switching capacitance. The simulation results shows that the Modified SRAM cell consumes less average power, which is $0.9 \mu \mathrm{W}$ for 1 Bit SRAM Cell and $1.79 \mathrm{~mW}$ for 16x16 Bit SRAM Cells.

Results show that the measured Delay is $0.04 \mathrm{~ns}$ for 1 Bit SRAM Cell which is having improvement up to $93 \%$, and is having a value of $0.03 \mathrm{~ns}$ for $16 \times 16$ Bit SRAM Cell Array.

Static Noise Margin value of Modified SRAM Cell is $434 \mathrm{mV}$. So, it is concluded that Modified SRAM cell can be used to provide Low Power solution in battery operated devices like mobile phones and laptops etc.

One can extend this work for higher frequency of operation also larger size of SRAM can be the future scope of this paper.

\section{REFERENCES}

[1] Singhal S., Gaur N., Mehra A. and Kumar P., "Analysis and comparison of leakage power reduction techniques in CMOS circuits," Signal Processing and Integrated Networks (SPIN), pp.936, 944, 19-20 Feb. 2015.

[2] Upadhyay P., Kundu P., Kar R., Mandal D. and Ghoshal S.P., "A novel 10T SRAM cell with low power dissipation in active and sleep mode for write operation," Computer Science and Software Engineering (JCSSE), pp.206,211, 14-16 May 2014.

[3] Leochico K. and John E., "Data retention voltage analysis of various low-power SRAM topologies," Circuits and Systems (MWSCAS), 2014 IEEE 57th International Midwest Symposium, pp.913,916, 3-6 Aug. 2014.

[4] Dhindsa, A.S and Saini S. "A novel differential 9t cell SRAM with reduced sub threshold leakage power," Advances in Engineering and Technology Research (ICAETR), pp.1, 5, 1-2 Aug. 2014.

[5] Sharma C.K. and Chandel R., "Analysis of SRAM cell designs for low power applications," Convergence of Technology (I2CT), pp.1, 4, 6-8 April 2014.

[6] Vanama K., Gunnuthula R. and Prasad G., "Design of low power stable SRAM cell," Circuit, Power and Computing Technologies (ICCPCT), pp.1263, 1267, 2021 March 2014.

[7] Singh C., Grover A. and Grover N., "Implementation of a Modified Model-SRAM Using Tanner EDA," Computational Intelligence, Modelling and Simulation (CIMSim), pp.390, 393, 24-25 Sept. 2013.

[8] Grover A. "Low Power 7-T SRAM Using $90 \mathrm{~nm}$ Technology with Tanner Tool," Artificial Intelligence, Modelling and Simulation (AIMS), pp.467, 470, 3-5 Dec. 2013

[9] Narayan S.L., Korah R. and Kumar N.K., "A novel sleepy stack 6-T SRAM cell design for reducing leakage power in submicron technologies," Communications and Signal Processing (ICCSP), 2013 International Conference, pp.753,757, 3-5 April 2013. 
[10] Akashe. S, Mishra. M and Sharma. S, "Self-controllable voltage level circuit for low power, high speed 7T SRAM cell at $45 \mathrm{~nm}$ technology," Engineering and Systems (SCES), 2012 Students Conference on , pp.1,5, 16-18, 2012.

[11] Jain A. and Sharma S., "Optimization of Low Power 7T SRAM Cell in 45nm Technology," Advanced Computing \& Communication Technologies (ACCT), pp.324, 327, 7-8 Jan. 2012.

[12] Yuan-yuan Wang, Zi-Ou Wang and Li-Jun Zhang, "A new 6-transistor SRAM cell for low power cache design," Solid-State and Integrated Circuit Technology (ICSICT), pp.1,3, Oct. 29 2012-Nov. 12012.

[13] Upadhyay P., Kar R., Mandal D. and Ghoshal S.P., "A low power CMOS voltage mode SRAM cell for high speed VLSI design," Microelectronics and Electronics (PrimeAsia), pp.25,28, 5-7 Dec. 2012.

[14] Teman A. and Fish, A., "Sub-threshold and nearthreshold SRAM design," Electrical and Electronics Engineers in Israel (IEEEI), pp.608,612, 17-20 Nov. 2010.
[15] Neil H. E. Weste, David Harris, Ayan Bannerjee ,CMOS VLSI Design - A Circuits and Systems Perspective, Pearson Education, 3rd Edition 2009.

[16] Keejong Kim, Mahmoodi H. and Roy K., "A LowPower SRAM Using Bit-Line ChargeRecycling," Solid-State Circuits IEEE Journal, vol.43, no.2, pp.446, 459, Feb. 2008.

[17] Sheng Lin, Yong-Bin Kim and Lombardi, Fabrizio, "A 32nm SRAM design for low power and high stability," Circuits and Systems, pp.422, 425, 10-13 Aug. 2008

[18] Park J.C. and Mooney V.J., "Sleepy Stack Leakage Reduction," Very Large Scale Integration (VLSI) Systems, IEEE Transactions, vol.14, no.11, pp.1250, 1263, Nov. 2006.

[19] Yen-Jen Chang, Feipei Lai and Chia-Lin Yang, "Zeroaware asymmetric SRAM cell for reducing cache power in writing zero," Very Large Scale Integration (VLSI) Systems, vol.12, no.8, pp.827,836, Aug. 2004.

[20] Sung-Mo Kang, Yusuf Leblebici, CMOS Integrated Circuits-Analysis and design, Tata McGraw-hill Edition, 3rd Edition 2005. 